\title{
Acquisition, Pointing, and Tracking
}

\author{
Mohammad A. Karim \\ University of Dayton \\ Center for Electro-Optics \\ 300 College Park Avenue \\ Dayton, Ohio 45469-0227
}

Electro-optical systems, both passive and active, eventually end up processing all kinds of signals. However, like all other crude jokes of life, the signal is never too obvious; it is often intertwined with corrupting noises. Thus, although the processing of signals is vital, much more occurs before such processing. The systems under consideration not only must acquire signals, but they also must recognize the sources of signals, locate such sources, and even track sources for uninterrupted acquisition. Although the research field that deals with these related but tricky issues historically seemed to involve only defense-related applications, it is now poised for many dual-use and commercial applications. This special section of Optical Engineering on acquisition, pointing, and tracking summarizes the work of 43 different researchers from government, academia, and industry. The 20 papers are fortuitously categorized into four subareas: acquisition systems, detection/recognition processes and systems, pointing/ tracking algorithms, and pointing/tracking support systems.

The first five papers deal with acquisition systems in general. In the first paper, Iftekharuddin and Karim use spatial averaging techniques to evaluate the acquisition performance of staring focal plane arrays as a function of their pixel geometries. Next, McManamon, Watson, Dorschner, and Barnes discuss the use of liquid crystal writable gratings for passive beam-steering applications in microscan systems. In addition to steering the field of view over a large field of regard, such writable gratings can achieve the same sensitivity as the existing linear scanning sensors. In the third paper, Watson describes the development of a beam-steering system by means of a cascade of decentered microlens arrays. In the next paper, Salisbury, McManamon, and Duncan describe the use of a rare-earth-doped optical fiber amplifier for increasing the SNR of a continuous-wave $1-\mu \mathrm{m}$ all-solidstate ladar system. Finally, in the fifth paper, Overbeck, Mark, McCracken, McManamon, and Duncan compare coherent and incoherent detection by means of an eyesafe 2.09. $\mu \mathrm{m}$ ladar system.
The next six papers deal with detection and recognition processes as well as systems. In the first paper, Rao presents a technique that is used to rank and extract salient contours from a 2-D image acquired by a passive sensor. In particular, this technique assigns a saliency measure to contours based on length, smoothness, and contrast. In the second paper, Auborn, Fuller, and McCauley use a co-occurrence matrix target detection algorithm as well as a suitably designed processor architecture for target segmentation in representative IR imagery. Next, Clayton, Driggers, and Harmon present a software simulation of a multiaperture vision system (with one, three, and nine eyelets) that locates a target by means of a back-propagation neural network. In the fourth paper, Karch, Karim, and Flannery study the impact of recognizing defocused images/patterns/targets (both in the absence and presence of noise) by means of a binary joint transform optical correlator. This study also compares the effects of using constant and adaptive thresholding in correlation. Next, Zhou, Zhao, Yu, and Chao study the impact of using a phase-difference prewhitening technique and show that it improves interclass multiobject discrimination. In the sixth paper, Perez and Karim modify a joint transform correlator by incorporating a logarithmic-polar transformation and use the resulting system to recognize scaled and rotated images/targets of interest.

The third group of papers, four in all, concerns pointing and tracking algorithms. In the first paper, Shetty and Alouani use a set of two detectors for a rather fast maneuver detection application without significantly increasing the memory requirement. In the second paper, Eeckman, Colvin, and Axelrod use a biologically inspired method consisting of five hexagonally packed layers of single compartment neurons for motion detection and tracking applications. Next, Skormin, Herman, Tascillo, and Nicholson present a mathematical model of a pointing, acquisition, and tracking system for application in laser-based intersatellite communication. Then, Skormin, Tascillo, and Nicholson introduce a self-tuning 
feedforward jitter compensation scheme to the mirror positioning control for de-emphasizing satellite jitter effects.

The final subarea consists of five papers that deal with the support systems used in pointing and/or tracking. First, Nowakowski and Simuoli interrelate the angular position and image quality of an active precision tracking system consisting of an agile scanning transmitter and a receiver. The effects of shot noise and beam jitter on angular position estimation are particularly explored in this work. Then, Awwal and Power use a complex associative memory based on a neural network architecture for tracking 3-D objects in dynamic environments. This scheme is readily amenable to optoelectronic implementation. Next, Khattak presents an innovative design of a refracting-type laser beam scanning device that exhibits a minimum across-scan error. In the fourth paper, Amat, Aranda, and Casals use a PC-driven hardware architecture for guiding a robot servo-positioning system to be used in commercial pointing and tracking applications. Finally, Bigley presents a control system stabilizing technique utilizing the differing characteristics of the active state variables for designing wideband stable platforms suitable for tracking and pointing applications.

In conclusion, this special section is well balanced and reports the ongoing research efforts on acquisition, pointing, and tracking at different government, university, and industrial laboratories. I would like to thank the many contributors and reviewers for their dedication. Without their help and timeliness, this special section would not have been possible.

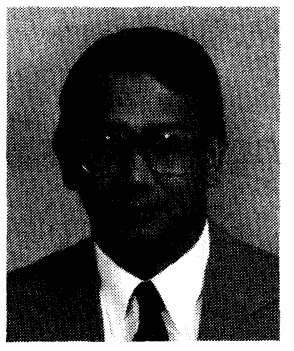

Mohammad A. Karim is the director of the Center for Electro-Optics and a professor in both the electrical engineering department and the electro-optics program at the University of Dayton (UD). He received his BS in physics in 1976 from the University of Dacca, Bangladesh, and MS degrees in physics and electrical engineering and $\mathrm{aPh}$ in electrical engineering from the University of Alabama in 1978, 1979, and 1981,

respectively. Dr. Karim's current research interests include pattern/target recognition, digital systems, optical system design, IR imaging systems, displays, optical phased array radar, beam agility and transformation, heterodyne detection, and optical computing. Dr. Karim is the author or coauthor of more than 160 published papers including 95 journal articles. He is the author of the graduate textbook Electro-Optical Devices \& Systems (1990), coauthor of two other textbooks, Digital Design: A Pragmatic Approach (1987) and Optical Computing: An Introduction (1992), and the editor of the reference book Electro-Optical Displays (1992). He was the guest editor of two other Optical Engineering special issues on electro-optical displays and IR imaging systems in 1990 and 1991, respectively. He is the guest editor of an upcoming Optics \& Laser Technology special issue on optical computing in 1994. Dr. Karim serves on the editorial boards of Microwave \& Optical Technology Letters and Optics \& Laser Technology. He is a Fellow of the Optical Society of America and is the faculty advisor of UD's student chapter of SPIE. In 1991, he won UD's highest award for scholarship. 